\title{
Rapid Development of Intracranial Aneurysm Associated with Tuberculous Meningitis
}

\author{
Hyunjo Lee, Taewon Kim
}

Keywords: Cerebral aneurysms, Meningitis

doi:10.1017/cjn.2017.229

Can J Neurol Sci. 2017; 44: 730-731

A 34-year-old woman presented to the emergency room (ER) with a headache and fever for 2 days. She denied any past medical history, and the blood and biochemical studies including human immunodeficiency virus antigen/antibody were unremarkable. Initial computed tomography (CT) of the brain was also unremarkable. She refused further evaluation, including lumbar puncture, and was discharged of her own will, against the opinion of the medical team. Six days later she revisited the ER with a worsened headache, fever, and dizziness. Her body temperature was $38^{\circ} \mathrm{C}$. A neurologic examination revealed gaze-evoked nystagmus beating toward the right side. Analysis of the cerebrospinal fluid (CSF) showed lymphocyte-dominant pleocytosis $(460 / \mu \mathrm{l})$, a decreased CSF/serum glucose ratio (0.4), elevated protein $(89.5 \mathrm{mg} / \mathrm{dl})$, and a normal value of adenosine deaminase (4 U/l). A CSF Gram stain, India ink test, and acid-fast bacilli (AFB) smear were all negative. Serologic tests-including cryptococcal antigen, anti-HIV, and VDRL-were also negative. The MRI demonstrated leptomeningeal enhancement around the basal and quadrigeminal cisterns (Figure 1a). The MRA was unremarkable (Figure 1b,c,d). Empirical antibiotics covering tuberculous meningitis (TBM) and dexamethasone were initiated under the diagnosis of probable TBM. ${ }^{1}$ Two days later she developed a generalized tonic-clonic seizure with continuous stupor mentality. The immediate follow-up CT angiography showed a subarachnoid hemorrhage (SAH; Figure 2a) with an aneurysm on the top of the basilar artery (Figure 2b,c,d), which had not been demonstrated two days prior. The diameter of the aneurysm was approximately $0.7 \mathrm{~cm}$ (Figure 3). An endovascular embolization with coiling was performed. Without clinical improvement, she died 1 month after admission.

Hydrocephalus and cerebral ischemia are commonly associated with TBM, but infectious intracranial aneurysms and subsequent $\mathrm{SAH}$ are rarely encountered. ${ }^{2,3}$ The autopsy study reported that arterial aneurysms were noted in only 2 cases among 51 postmortem TBM brains. ${ }^{2}$ Tuberculous aneurysms have also been reported in other extracranial vessels, such as the thoracic and abdominal aorta. ${ }^{4}$

The unique feature of this case is the rapidity of formation of the aneurysm. A de-novo cerebral aneurysm developing as rapidly as within 2 days, as in our case, has not yet been reported. Clinicians should be warned to be aware of a potential rapid progression of aneurysmal formation and subsequent SAH in TBM.
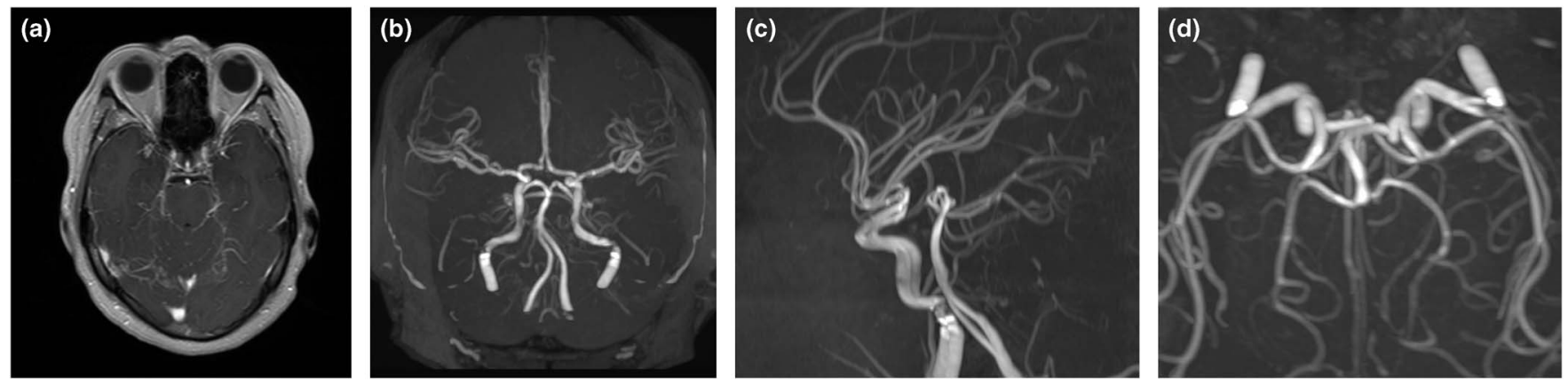

Figure 1: Initial MRI and MR angiography of the brain demonstrates leptomeningeal enhancement around the basal and quadrigeminal cisterns on the contrast-enhanced T1-weighted image (a), with unremarkable intracranial vessels; $(b)$ anterior-posterior view; $(c)$ left-lateral view; $(d)$ inferiorsuperior view.

From the Department of Neurology, Incheon St. Mary's Hospital, College of Medicine, The Catholic University of Korea, Incheon, Republic of Korea.

Received February 20, 2017. Final Revisions Submitted May 1, 2017. Date of Acceptance June 19, 2017.

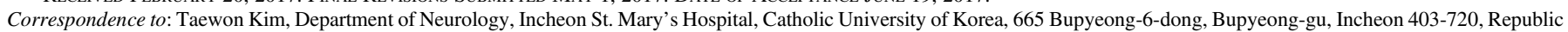
of Korea. Email: kimtaewon@ catholic.ac.kr 

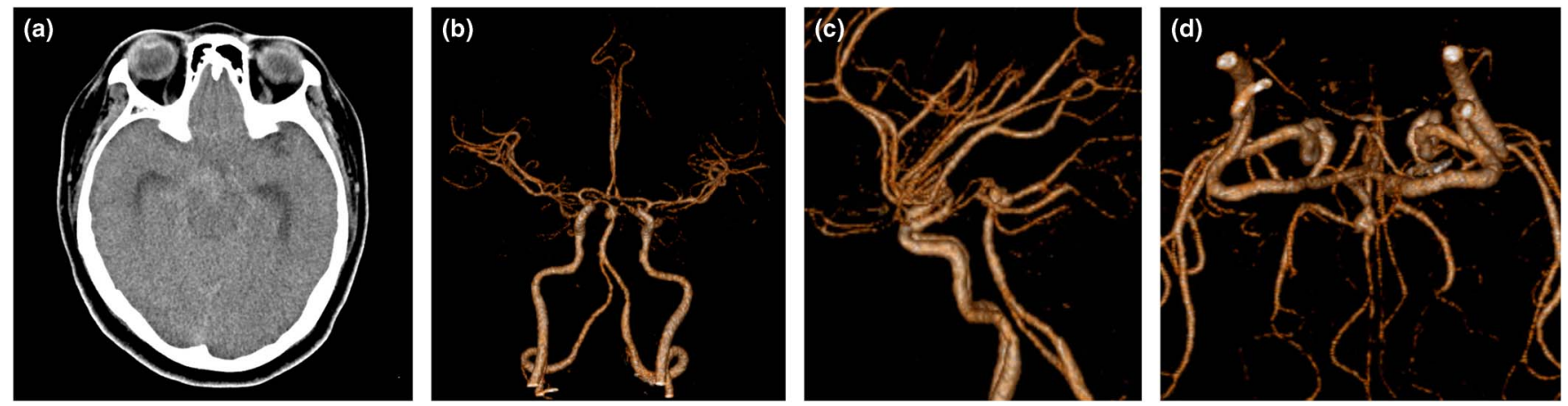

Figure 2: The follow-up CT two days later demonstrates SAH: (a) with an aneurysm on the top of the basilar artery; (b) anterior-posterior view; (c) left-lateral view; (d) inferior-superior view.
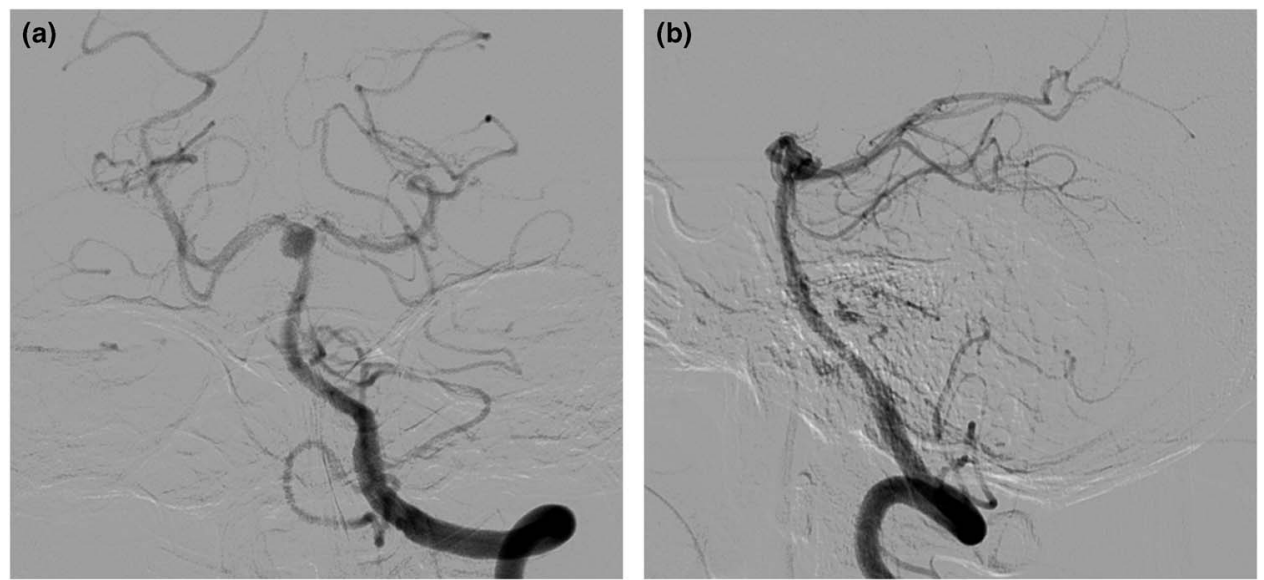

Figure 3: Digital subtraction angiography shows an approximately 7-mm sized aneurysm on the top of the basilar artery.

\section{DisClosures}

Hyunjo Lee and Taewon Kim hereby declare that they have nothing to disclose.

\section{REFERENCES}

1. Thwaites G, Fisher M, Hemingway C, et al. British Infection Society guidelines for the diagnosis and treatment of tuberculosis of the central nervous system in adults and children. J Infect. 2009; 59(3):167-87.
2. Chatterjee D, Radotra BD, Vasishta RK, Sharma K. Vascular complications of tuberculous meningitis: an autopsy study. Neurol India. 2015;63(6):926-32.

3. Roh JH, Kwon DY, Park MH. A case of intracranial aneurysm and subarachnoid hemorrhage with tuberculous meningitis. Neurol Asia. 2011;16(2):157-61.

4. Long R, Guzman R, Greenberg H, Safneck J, Hershfield E. Tuberculous mycotic aneurysm of the aorta: review of published medical and surgical experience. Chest. 1999; 115(2):522-31. 